\title{
Indoor Location Algorithm in Complex Wireless Environment
}

\author{
Zhangang Wang ${ }^{1, \text { a }}$, Zhiyu Lu, , , Hang Lu, ${ }^{1, b}$, Yankui Zhang ${ }^{1, \mathrm{c}}$ \\ and Donghang Jia ${ }^{1, d}$ \\ ${ }^{1}$ Information Engineering University, Zhengzhou 450001, China; \\ a313903150@qq.com, b1421401588@qq.com, '834799435@qq.com, ${ }^{\text {d }}$ 'Licinso1991@gmail.com
}

Keywords: 3-dimensional positioning, TDOA, Two-Step Least Squares(TSLS), unscented Kalman filter (UKF), line-of-sight(LOS), non-line-of-sight(NLOS)

\begin{abstract}
Aiming at the 3-dimensional positioning problem in the indoor wireless environment, TDOA-based localization model is presented in this paper. The Two-Step Least Squares(TSLS) and unscented Kalman filter (UKF) algorithm are used to solve the target's position. In the case that the line-of-sight(LOS) or non-line-of-sight (NLOS) environment is unknown, the algorithm can adaptively identify the environmental parameters, and can give the accurate positioning results of the target. The positioning accuracy of the algorithm can reach decimeter level, so the algorithm meets the need of the indoor positioning better.
\end{abstract}

\section{Introduction}

With the rapid development of wireless communication network and mobile Internet, the location based service (LBS) has become one of the most promising and potential services. Although commercial GPS has been widely used with the development of smart phones, its performance is poor in such as indoor, underground, high-rise urban areas and many other scenes. Since the positioning system based on the wireless network base station has advantages over the GPS in coverage breadth and depth, it gets more and more attention from operators.

In the positioning technique based on a wireless communication base station, as the impact of shelter in the process of transmission, the propagation environment is divided into line-of-sight propagation(LOS) environment and non-line-of-sight propagation(NLOS) environment. In the NLOS environment[1], the signal's propagation is blocked and it leads to the signal through other paths to reach the base station, increases the reception time of the signal, then the performance of positioning by Time of Arrival(TOA) has been affected. Therefore, the establishment of a good measurement model has a greater impact on time difference of arrival(TDOA) positioning accuracy.

In order to establish the measurement model of the signal, the test data are analyzed firstly. The NLOS model is established as the multiplicative model, that is, the time of arrival under the NLOS environment is $k$ times of the time of arrival directly. The existence of the parameter $k$ makes the localization equation show a more serious non-linear characteristics, so the least squares algorithm can't be directly used to solve the problem. In order to estimate the parameter $k$, the UKF algorithm[2] is introduced to estimate the position of the target and the parameter $k$ in this paper. Although the UKF can estimate the target location, but the calculation of UKF is complex and time-consuming[3], so it is not suitable for use on a large scale. In order to solve this problem, a two-step localization strategy is proposed. First, the NLOS environment parameter $k$ is estimated by the UKF algorithm, and then the Two-Step Least Squares algorithm[4] is used to locate the coordinates quickly. In the problem, since the time between the target and the base station may not be synchronized, TDOA[5] is used in this paper. The influence of synchronization error can be eliminated by using time difference to locate. After obtaining the positioning result, the error of it is analyzed and the method of error correction in practical situation is given. The results show that the positioning accuracy of the algorithm can reach centimeter level in $\mathrm{x}$, $\mathrm{y}$ coordinates and decimeter level in z coordinate. 


\section{Model establishment}

\subsection{Environmental measurement model}

The time delay of the Mobile Station (MS) to the Base Station (BS) is increased through the NLOS transmission. For different environmental conditions, the influence to time delay by NLOS transmission is different. General error in the time delay caused by NLOS transmission can be built by additive, multiplicative, exponential distribution, uniform distribution and so on. So the positioning model for single MS can be established as follows:

$$
\begin{aligned}
& D=\sqrt{\left(x_{i}-x_{j}\right)^{2}+\left(y_{i}-y_{j}\right)^{2}+\left(z_{i}-z_{j}\right)^{2}}=c t_{j}^{i} \\
& \bar{D}=k D+c \tau_{i}+n
\end{aligned}
$$

Where $x_{i}, y_{i}, z_{i}$ is the real coordinates for the $i$-th MS, $x_{j}, y_{j}, z_{j}$ is the real coordinates for the $j$ -th BS, $k$ is the proportional coefficient of time delay caused by NLOS transmission, $t_{j}^{i} \in T$ is the measured time delay from the $i$-th MS to the $j$-th BS, $n$ is the measurement error caused by measurement equipment, environmental noise and so on, $\tau_{i}$ is the synchronous time error between the $i$-th MS and every BS. Considering the synchronous time error is the same for the MS to each BS, TDOA-based positioning method is used to offset the effect of synchronous time error on the positioning by the difference of signal time delay from MS to BS. The principle of TDOA-based localization method is introduced as follow.

\subsection{TDOA location principle}

When the distance difference between the base station $B S_{i}(i=1,2, \ldots)$ and the MS is known as $d_{21}=d_{2}-d_{1}$, MS must be located in the linear hyperbolic which take the two BS as the focus, and make the distance difference to the focus constant for $d_{21}$, as shown in Fig.1. Thus, the intersection of the two sets of hyperbolas realizes the estimation of the MS position.

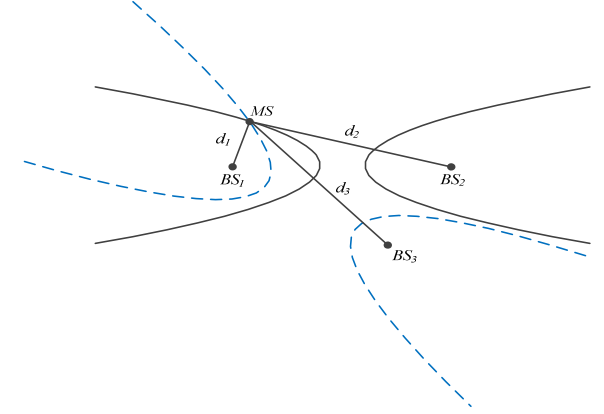

Fig. 1 The model of TDOA location

Let $\left(x_{i}, y_{i}\right)$ be the estimated position of the i-th MS and $\left(x_{j_{1}}, y_{j_{1}}\right)$ be the known position of the $\mathrm{BS}$, so the distance between the i-th MS and the $j_{1}$-th BS is given by:

$$
d_{j_{1}}^{i}=\sqrt{\left(x_{j_{1}}-x_{i}\right)^{2}+\left(y_{j_{1}}-y_{i}\right)^{2}}
$$

Let the distance difference between the i-th MS and the $j_{2}$-th BS be $d_{j_{2}}^{i}$, the delay of the signal arriving at the two BSs is:

$$
d_{j_{1}, j_{2}}^{i}=d_{j_{1}}^{i}-d_{j_{2}}^{i}=c\left(t_{j_{1}}^{i}-t_{j_{2}}^{i}\right)=\sqrt{\left(x_{j_{1}}-x_{i}\right)^{2}+\left(y_{j_{1}}-y_{i}\right)^{2}}-\sqrt{\left(x_{j_{2}}-x_{i}\right)^{2}+\left(y_{j_{2}}-y_{i}\right)^{2}}
$$

Where $c$ is the wave propagation velocity, $t_{j_{1}, j_{2}}^{i}=t_{j_{1}}^{i}-t_{j_{2}}^{i}$ is the TDOA measurement. Finally, according to the data obtained by multiple measurements, the MS's coordinates can be calculated of the value $\left(x_{i}, y_{i}\right)$. 


\section{Positioning algorithm}

\subsection{Estimation of the NLOS Environment Parameters Based on Unscented Kalman Filter (UKF)}

On the basis of the above-mentioned measurement model, the position coordinates of the mobile terminals in different scenes are solved. In the same scene, the proportional coefficient $k$ is approximately equal; for different scenarios, $k$ is different. If $k$ is estimated as an unknown quantity, the estimation equation will exhibit severe non-linear characteristics. In order to solve this problem, this paper firstly estimates the delay measurement data $k$ using the UKF algorithm and the delay measurement data of the first $p$ MSs. After $k$ is known, in order to reduce the positioning time-consuming, and then using two-step least square(TSLS) algorithm to estimate the location of all mobile terminals.

Using the UKF algorithm, the system state equation and observation equation are established as follows:

$$
\left\{\begin{array}{l}
X_{t}=X_{t-1}+u_{t-1} \\
Z_{t}=h\left(X_{t}\right)+v_{t}
\end{array}\right.
$$

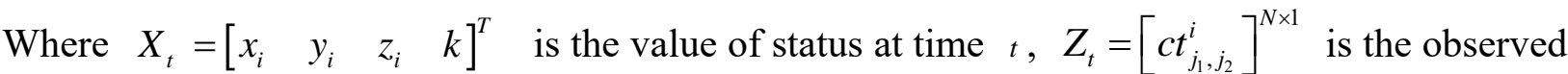
values at time $t$ which length is $N . h\left(X_{t}\right)=k \cdot \sqrt{\left(x_{i}-x_{j}\right)^{2}+\left(y_{i}-y_{j}\right)^{2}+\left(z_{i}-z_{j}\right)^{2}}$ is the observation model, $u_{t}$ and $v_{t}$ are state noise and observation noise, respectively, which obey Gaussian distribution. The algorithmic flow is as follows:

a. Initial setting

Set the initial value of the value of status and its covariance matrix $X_{0}, P_{0}$, for $t=0$, the value of status and its covariance matrix are:

$$
\begin{aligned}
& \hat{X}_{0}=E\left[X_{0}\right] \\
& P_{0}=\operatorname{COV}\left[X_{0}\right]
\end{aligned}
$$

b. Calculation of point sigma

Calculate the point sigma of the state value at time $t-1$

$$
\begin{aligned}
& \chi_{0, \mathrm{t}-1}=\hat{X}_{t-1} \quad W_{0}=\kappa /(n+\kappa) \\
& \chi_{i, \mathrm{t}-1}=\hat{X}_{t-1}+\left(\sqrt{(n+\kappa) P_{t-1}}\right)_{i} \quad W_{i}=1 / 2(n+\kappa) \\
& \chi_{i+n, t-1}=\hat{X}_{t-1}-\left(\sqrt{(n+\kappa) P_{t-1}}\right)_{i} \quad W_{i+n}=1 / 2(n+\kappa)
\end{aligned}
$$

Where $\left(\sqrt{(n+\kappa) P_{t-1}}\right)_{i}$ denotes the $i$-th row of $\left(\sqrt{(n+\kappa) P_{t-1}}\right)_{i}, \kappa$ is a constant value and $W$ denotes a weight.

c. Time update

The point sigma at time $t$ is calculated from the point sigma at time $t-1$ :

$$
\chi_{i, t}=\chi_{i, t-1}+u_{t-1}
$$

The observations of point sigma at time $t$ can be calculated by the point sigma at time $t-1$ :

$$
\mathbb{Z}_{i, t}=h\left(\chi_{i, t-1}\right)+v_{t}
$$

One-step prediction of the state value at time $t$ can be calculated by the point sigma at time $t$ :

$$
\hat{X}_{\mathrm{t} \mid \mathrm{t}-1}=\sum_{i=0}^{2 n} W_{i} \chi_{i, t}
$$


Calculating the one-step prediction matrix of the covariance matrix of state value at time $t$ :

$$
P_{k \mid t-1}=\sum_{i=0}^{2 n} W_{i}\left\{\chi_{i, t}-\hat{X}_{t \mid t-1}\right\}\left\{\chi_{i, t}-\hat{X}_{t \mid t-1}\right\}^{T}+Q
$$

Calculating the one-step prediction of the system observations at time $t$ :

$$
\hat{Z}_{t \mid t-1}=\sum_{i=0}^{2 n} W_{i} \mathbb{Z}_{i, t}
$$

\section{d. Measurement updates}

Calculating the covariance matrix of the observations at time $t$ :

$$
P_{Z Z}=\sum_{i=0}^{2 n} W_{i}\left\{\mathbb{Z}_{i, t}-\hat{Z}_{t \mid t-1}\right\}\left\{\mathbb{Z}_{i, t}-\hat{Z}_{t \mid t-1}\right\}^{T}+R
$$

Calculating the covariance matrix of the observations and the state value at time $t$ :

$$
P_{X Z}=\sum_{i=0}^{2 n} W_{i}\left\{\chi_{i, t}-\hat{X}_{t \mid t-1}\right\}\left\{\mathbb{Z}_{i, t}-\hat{Z}_{t \mid t-1}\right\}^{T}
$$

Calculating the gain in the filter

$$
K=P_{X Z} P_{Z Z}^{-1}
$$

Calculating the estimated value of the system state value at time $t$

$$
\hat{X}_{t}=\hat{X}_{t \mid t-1}+K\left(Z_{t}-\hat{Z}_{t \mid t-1}\right)
$$

The covariance matrix of the system state value at time $t$ is obtained:

$$
P_{t}=P_{t \mid t-1}-K P_{Z Z} K^{T}
$$

According to the problem description, whether NLOS exists is unknown, so it can be determined by $k$, when $k=1$, there is no NLOS.

\subsection{Position Estimation Based on Two-step Least Squares}

When positioning based on TDOA, according to the results of multi-station measurements, Two-step least-squares algorithm is used to solve this problem, the process is as follows:

According to equation (2), we can get

$$
\left(d_{j_{1}}^{i}\right)^{2}=\left(x_{j_{1}}-x_{i}\right)^{2}+\left(y_{j_{1}}-y_{i}\right)^{2}=K_{j_{1}}-2 x_{j_{1}} x_{i}-2 y_{j_{1}} y_{i}+x_{i}^{2}+y_{i}^{2}
$$

Where $K_{j_{1}}=x_{j_{1}}{ }^{2}+y_{j_{1}}{ }^{2}$.

And according to equation (3), we can get

$$
\left(d_{j_{1}}^{i}\right)^{2}=\left(d_{j_{1}, j_{2}}^{i}+d_{j_{2}}^{i}\right)^{2}
$$

Combining equation (3) (4) (5), the equation can be get as follow:

$$
\left(d_{j_{1}, j_{2}}^{i}\right)^{2}+2 d_{j_{1}, j_{2}}^{i} d_{j_{2}}^{i}=K_{j_{1}}-2 x_{j_{1}, j_{2}} x_{i}-2 y_{j_{1}, j_{2}} y_{i}-K_{j_{2}}
$$

Where $x_{j_{1}, j_{2}}=x_{j_{1}}-x_{j_{2}}, y_{j_{1}, j_{2}}=y_{j_{1}}-y_{j_{2}}$.

The relationship between the terminal positions $\left(x_{i}, y_{i}\right)$ and $d_{j_{2}}^{i}$ can be obtained by solving the system of linear equations (19) by taking $x_{i}, y_{i}$ and $d_{j_{2}}^{i}$ as unknowns. The method is as follows:

The initial solution is obtained by applying the weighted least square estimation to the linear system of equations (19), and then using the known constraints such as initial solution and additional variables construct a new system of linear equations. Finally the weighted least squares 
estimation algorithm is applied to the new system to obtain a more accurate estimation position.

Let $z_{a}=\left[z_{p}^{T}, d_{j_{2}}^{i}\right]^{T}$ be an unknown vector, where $z_{p}=\left[x_{i}, y_{i}\right]^{T}$, from equation (19) we can get the error vector equation with TDOA noise as:

$$
\psi=h-G_{a} z_{a}^{0}
$$

Where $\psi$ is the error vector, $h=\frac{1}{2}\left[\begin{array}{c}\left(d_{2,1}^{i}\right)^{2}-K_{2}+K_{1} \\ \left(d_{3,1}^{i}\right)^{2}-K_{3}+K_{1} \\ \vdots \\ \left(d_{M, 1}^{i}\right)^{2}-K_{M}+K_{1}\end{array}\right], \quad G_{a}=\left[\begin{array}{ccc}x_{2,1} & y_{2,1} & d_{2,1} \\ x_{3,1} & y_{3,1} & d_{3,1} \\ \vdots & \vdots & \vdots \\ x_{M, 1} & y_{M, 1} & d_{M, 1}\end{array}\right]$, the results of weighted least squares estimation are:

$$
z_{a}=\left(G_{a}^{T} \Psi^{-1} G_{a}\right) G_{a}^{T} \Psi^{-1} h
$$

Where $\Psi$ is the covariance matrix of the error vector $\Psi=c^{2} B Q B, Q$ is the covariance matrix of the TDOA measurements, $B=\operatorname{diag}\left\{\left(d_{2}^{i}\right)^{0},\left(d_{3}^{i}\right)^{0}, \cdots,\left(d_{M}^{i}\right)^{0}\right\}$, and $\left(d^{i}\right)^{0}$ can be determined by $z_{a} \approx\left(G_{a}^{T} Q^{-1} G_{a}\right) G_{a}^{T} Q^{-1} h$

Constructing the linear system of equations:

$$
\psi^{\prime}=h^{\prime}-G_{a} z_{a}^{\prime}
$$

Where $\psi^{\prime}$ is the error vector of $z_{a}, h^{\prime}=\left[\begin{array}{c}\left(z_{a, 1}-x_{1}\right)^{2} \\ \left(z_{a, 2}-x_{2}\right)^{2} \\ z_{a, 3}^{2}\end{array}\right], G_{a}{ }^{\prime}=\left[\begin{array}{ll}1 & 0 \\ 0 & 1 \\ 1 & 1\end{array}\right], z_{a}{ }^{\prime}=\left[\begin{array}{c}\left(x_{i}-x_{1}\right)^{2} \\ \left(y_{i}-y_{1}\right)^{2}\end{array}\right]$. So the result of the weighted least squares estimation of equation (22) is:

$$
z_{a}{ }^{\prime}=\left(G_{a}^{\prime T} \Psi^{1^{\prime-1}} G_{a}^{\prime}\right) G_{a}^{\prime T} \Psi^{\prime^{\prime-1}} h^{\prime}
$$

Where $\Psi^{\prime}$ is the covariance matrix of $\psi^{\prime}, \Psi^{\prime}=4 B^{\prime} \operatorname{cov}\left(z_{a}\right) B^{\prime}, \operatorname{cov}\left(z_{a}\right)$ is the covariance matrix of $z_{a}, \operatorname{cov}\left(z_{a}\right)=\left(G_{a}^{T} \Psi^{-1} G_{a}\right)^{-1}, \quad B^{\prime}=\operatorname{diag}\left\{x_{i}^{0}-x_{1}, y_{i}^{0}-y_{1},\left(d_{1}^{i}\right)^{0}\right\}$.

The final target position is calculated as:

$$
z_{p}=\sqrt{z_{a}^{\prime}}+\left[\begin{array}{l}
x_{1} \\
y_{1}
\end{array}\right] \text { or } z_{p}=-\sqrt{z_{a}^{\prime}}+\left[\begin{array}{l}
x_{1} \\
y_{1}
\end{array}\right]
$$

In summary, the algorithmic Flow is summarized as follow:

1). Based on the TDOA localization principle, the UKF algorithm is used to estimate the NLOS environment parameters $k$;

2). The MS position coordinates are estimated based on the Two - Step Least Squares algorithm.

3). Position correction based on prior information

\section{Algorithm evaluation}

The test data are in the appendix, including the coordinates of the MSs and the coordinates of the BSs of the different locations in the five different scenes.

a. Solution of NLOS Environment Parameters $k$ Based on UKF Algorithm

The UKF algorithm is used to estimate the NLOS parameters of the simulated scenarios. The value of parameter $k$ is taken as 1.4146 , The estimation process is shown in Figure 2: 


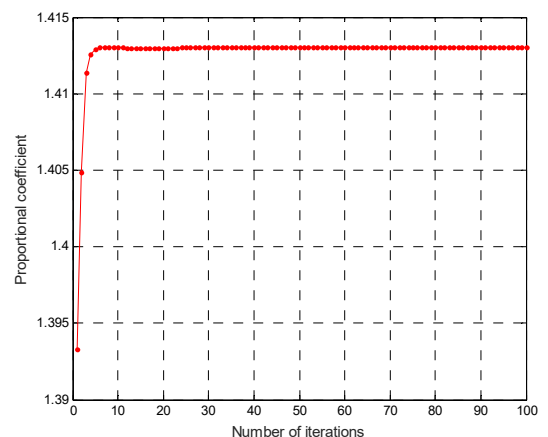

Fig. 2 Change of state quantity of UKF algorithm

It can be seen from the figure that after a few iterations of the UKF algorithm, the scale $k$ and position coordinates are able to converge quickly, the estimated result of parameter $\mathrm{k}$ is 1.4132 . The error between the $k$ that estimated by the UKF algorithm and the actual value of $k$ is very small. It is shown that the $k$ that estimated by the UKF algorithm can reflect the error caused by the NLOS.

b. Algorithm's Location Performance Verification

The UKF and the Two-Step Least Squares algorithm are used to locate the MSs, the localization error's distribution is shown in Figure 3. The three colors in the figure represent the positioning error of $x, y, z$ coordinates, respectively. It can be seen from the Figure 3 that the positioning error of $x, y$ are less than 0.5 meters, while the $\mathrm{z}$-coordinate positioning error is large.

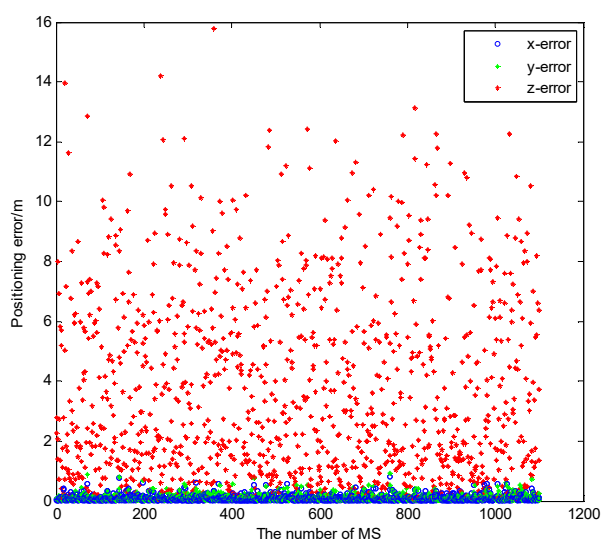

Fig.3 Positioning error distribution of case 1

\section{Error Analysis and Correction of Results}

In order to analyze the sources of the error in Figure 3, we discuss the performance bounds based on the Two-Step Least Squares algorithm in solving the TDOA localization model. The number of base stations is 30 .

Figure 4 shows the location of the BS on different planes in space. It can be seen from the coordinates of the BS that the $\mathrm{Z}$ coordinate's range is small relative to the XY's coordinate. Whether the three-dimensional positioning accuracy of the target is affected in this case is worthy of study. 

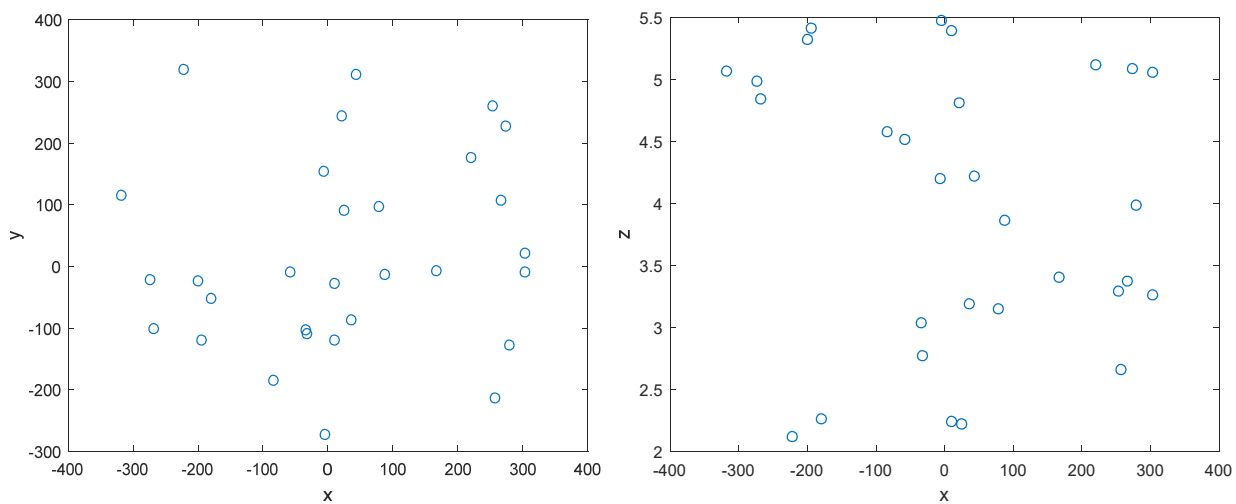

Fig.4 The position of the BS on different plane of space

In order to explore whether the Z-coordinate that is too concentrated will have a greater impact on the positioning accuracy, reference [6] and [7] for the discussion of the positioning accuracy of the Cramerian bound, the result shown in Fig. 6 can be obtained. Where WLS_1 and CRLB_1 represent the simulation results when the original target data is located on the first target, WLS_10 and CRLB_10 represent the simulation results of the original target data when the Z- coordinate is enlarged by 10 times. It can be seen from the figure that if the distance of the $Z$-coordinate enlarged by 10 times, the positioning accuracy is improved obviously.

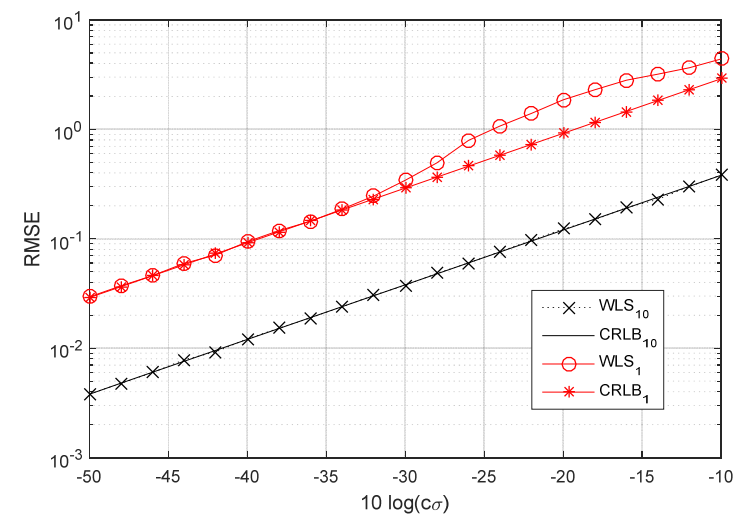

Fig.5 The curve of the positioning mean square error as the noise changes

Both the simulation results and the actual test results show that the positioning results have a greater impact when the XY coordinate is larger, the Z-coordinate is more concentrated, and the estimation precision of the Z-coordinate will become very low. Therefore, it can also be concluded that in the case of three-dimensional positioning, the distribution of base stations should be distributed, try not to coplanar or collinear.

Through the above analysis, we can see that even if only affected by small noise, the estimation error of the z-coordinate may also be too large. In order to meet the needs of the actual positioning accuracy, the proposed algorithm corrects the result of z-coordinate. Taking into account that the $\mathrm{z}$-coordinate of the hand-held MS is located within 1-2 meters, for the estimated value that beyond this limit, the $\mathrm{z}$ coordinate is corrected by 1.5 meters.

It can be seen from Table. 1 that the algorithm can estimate the position of MS more accurately in different scenes, and the effectiveness of the proposed algorithm is proved.

Table.1. Average positioning error of different case

\begin{tabular}{cccccc}
\hline Error $/ \mathrm{m}$ & Scene 1 & Scene 2 & Scene 3 & Scene 4 & Scene 5 \\
\hline $\mathrm{x}$ & 0.0948 & 0.0936 & 0.0683 & 0.0670 & 0.1433 \\
$\mathrm{y}$ & 0.1062 & 0.0966 & 0.0601 & 0.0643 & 0.1416 \\
$\mathrm{z}$ & 0.2570 & 0.2517 & 0.2538 & 0.2589 & 0.2542 \\
\hline
\end{tabular}

\section{Summary}

This paper solves the problem of indoor location in complicated wireless environment. Based on 
the measured data, the channel propagation model in complex wireless environment is analyzed, and the paper establishes the measurement model of NLOS. The measurement model can be adapted to the LOS and NLOS environments. Then TDOA-based localization method is proposed to solve the problem of delay localization in the presence of synchronization errors. In order to estimate the measurement model parameters, a parameter estimation method based on UKF is proposed and it can get high-precision NLOS environment parameters. Although UKF can also estimate the coordinates of the BS, it has high computational complexity. So the paper uses the thinking of two-step method. After obtaining the parameter $k$ of the model, it uses the two-step least square estimation method to solve the target position, the computational complexity of it has been reduced. Simulation results show that the proposed method's positioning accuracy can reach decimeter level, so it is an excellent localization algorithm for indoor positioning.

\section{References}

[1] Huang Y, Zhang S, Jing Y. An indoor mobile localization strategy based on particle filter in NLOS environment[C]// Chinese Control and Decision Conference. IEEE, 2016.

[2] Yu Liu, Hong Wang and Chaohuan Hou. UKF Based Nonlinear Filtering Using Minimum Entropy Criterion[J], IEEE Transactions on Signal Processing, 61(20): 4988-4989,2013.

[3] Lubin Chang, Baiqing $\mathrm{Hu}, \mathrm{An} \mathrm{Li}$, and Fangjun Qin. Transformed Unscented Kalman Filter[J], IEEE Transactions on Automatic Control, 58(1):252-257,2013.

[4] Shi Z, Feng Z. A new array pattern synthesis algorithm using the two-step least-squares method[J]. Signal Processing Letters IEEE, 2005, 12(3):250-253.

[5] Ho K C, Sun M. Passive Source Localization Using Time Differences of Arrival and Gain Ratios of Arrival[J] ,IEEE Transactions on Signal Processing , 56(2):464-477, 2008.

[6] Ho K C, Xu W W. An Accurate Algebraic Solution for Moving Source Location Using TDOA and FDOA Measurements[J]. IEEE Transactions on Signal Processing, 52(9): 2453-2463,2004.

[7] K. C. Ho, Xiaoning Lu, and L. Kovavisaruch. Source Localization Using TDOA and FDOA Measurements in the Presence of Receiver Location Errors: Analysis and Solution[J]. IEEE Transactions on Signal Processing, 55(2):684-696,2007. 